\title{
ON THE DIFFERENTIAL GEOMETRY OF CLOSED SPACE CURVES
}

\section{WERNER FENCHEL}

1. The subject of this lecture, the study of the differential geometric properties of a space curve which depend on the assumption that the curve is closed, is a rather modest chapter of the differential geometry in the large. The results are often comparatively elementary and seem to be isolated. On the other hand, the intuitive character of the statements and the lack of a general method of approach make the field attractive, and in the latest years several authors have contributed to it. Therefore, it might be justified to give a survey of its actual state. In an attempt to gather some results and problems from a common point of view it turned out that the differential geometry of space curves becomes more satisfactory if it is developed under slightly weaker assumptions than those usually adopted. Furthermore it proves to be useful to attach more importance to the simple geometrical relations between the spherical indicatrices of a curve and to the kinematical interpretation initiated by G. Darboux. Though many of the results are or can be generalized to curves in a euclidean space of arbitrary dimension, the following exposition is restricted to the case of the ordinary space, and it will not be possible to mention all contributions to the subject.

2. Let $s, 0 \leqq s \leqq l$, denote the arc length and $\boldsymbol{r}(s)$ the position vector of a variable point of a space curve $K$. Differentiation with respect to $s$ is indicated by a prime. Our assumptions are the following: The coordinates of $\boldsymbol{r}(s)$ are functions of class 4. To every point $\boldsymbol{r}(s)$ is attached an osculating plane, that is, a plane containing the vectors $\boldsymbol{t}=\boldsymbol{r}^{\prime}$ and $\boldsymbol{r}^{\prime \prime}$ such that its suitably oriented normal unit vector $\boldsymbol{b}(s)$, the binormal vector of $K$, has coordinates which are functions of class $2 .{ }^{1}$ The vectors $t^{\prime}$ and $b^{\prime}$ do not vanish simultaneously. For the sake of simplicity we further assume that they vanish only at a finite number of points. This implies that no arc of $K$ is contained in a plane.

By this formulation we avoid the usual assumption that $\boldsymbol{r}^{\prime \prime}$ and,

An address delivered before the Berkeley Meeting of the Society on April 29, 1950 , by invitation of the Committee to Select Hour Speakers for Far Western Sectional Meetings; received by the editors May 18, 1950.

${ }^{1}$ Actually we are given an osculating strip in the sense of Blaschke [1, p. 72]. (Numbers in brackets refer to the references at the end of the paper.) 
hence, the curvature never vanish. Define now the principal normal vector by

$$
n=b \times t
$$

( $X$ denoting the vector product) and the curvature $\kappa$ and the torsion $\tau$ by

$$
t^{\prime}=\kappa n, \quad b^{\prime}=-\tau n
$$

which is possible since these derivatives are perpendicular to both $t$ and $\boldsymbol{b}$. From the definition of $\boldsymbol{n}$ follows

$$
n^{\prime}=-\kappa t+\tau b .
$$

Hence Frenet's formulae are valid in the usual form, but here the curvature may vanish and even be negative. Of course, only a change of the sign of $\kappa$, not the sign itself, has a geometrical meaning; for, $\boldsymbol{b}$ (for all $s$ ) and, hence, $\boldsymbol{n}$ may be replaced by the opposite vectors. From the above assumptions we have

$$
\omega=\left(\kappa^{2}+\tau^{2}\right)^{1 / 2}>0 .
$$

A point of $K$ where either $\kappa$ or $\tau$ changes sign is called a point of inflection. If necessary we distinguish between $\kappa$-inflections and $\tau$ inflections. The number of these points is finite.

3. If the trihedral $t, b, n$ is laid off from the origin $O$, the vectors $t, b$, and $n$ describe three curves $T, B$, and $N$, the spherical indicatrices of the tangent, of the binormal, and of the principal normal of $K$. With $s$ considered as the time, the motion of the trihedral is a rotation about $O$ with the angular velocity $\omega=\omega(s)$ and an instantaneous axis of rotation which must lie in the plane $b, t$, because it is perpendicular to the velocity vectors (2). Let $\mathrm{c}$ be the unit vector on this axis such that its sense together with the sense of the rotation form a right-handed screw. Since $\mathrm{c}$ is perpendicular to the velocity vector (3) we then have

$$
\omega c=n \times n^{\prime}=\kappa b+\tau t .
$$

Let $\phi=\phi(s)$ denote the angle from $b$ to $c$ measured in the sense of the shortest rotation which brings $b$ into $t$. Then we have

$$
\kappa=\omega \cos \phi, \quad \tau=\omega \sin \phi .
$$

Besides the fixed unit sphere $S$, consider a moving unit sphere $S^{*}$ with center $O$ rigidly attached to the trihedral. The two loci of the instantaneous center of rotation on $S$ and on $S^{*}$ respectively are called the fixed centrode and the moving centrode of the motion of $S^{*}$. It 
is well known that this motion is obtained by letting the moving centrode roll on the fixed centrode. In our case the fixed centrode is the curve $C$ described by $\mathrm{c}=\mathrm{c}(s)$ on $S$ and the moving centrode is the great circle determined by $t$ and $b$. Hence the motion of the trihedral about $O$ is obtained by letting this great circle roll on $C$. From this it follows that the indicatrices $T$ and $B$ are spherical involutes of $C$. They are described by two points of the rolling great circle which have the spherical distance $\pi / 2$ (Fig. 1).

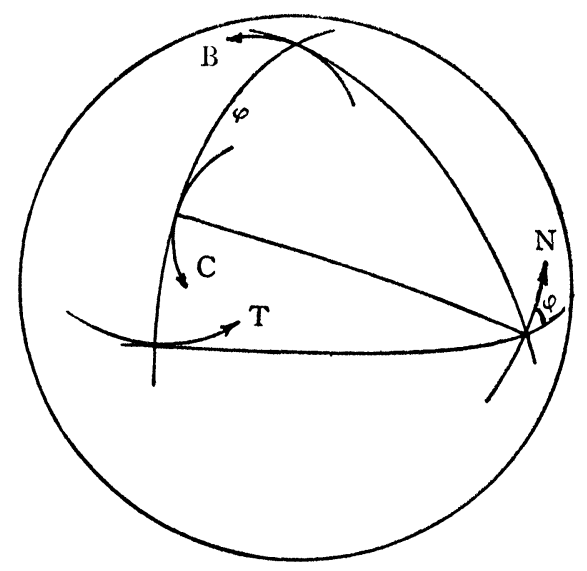

FIG. 1

4. A line element on the unit sphere is by definition a pair consisting of a point on $S$ and an oriented great circle through this point. The spherical polarity is an involutory mapping of the set of all line elements onto itself which maps an oriented great circle on that one of its poles which lies on its left-hand side seen from the exterior of the sphere. To every spherical curve-considered as the set of its line elements, point and tangent great circle-corresponds a unique polar curve, and this correspondence is involutory. The tangent vectors at corresponding points of two polar curves are opposite. To a point of inflection of one of the curves corresponds a cusp of the first kind of the other. In order to preserve complete duality we take the arc length on a spherical curve with a sign in such a way that the differential of the arc changes sign at a cusp. The infinitesimal length of the arc between two neighboring points of a curve then equals the angle between the corresponding tangent great circles of the polar curve, this angle being taken with a sign in the usual way. From this it follows that polar curves have reciprocal geodesic curvatures.

(1) and (2) yield that $T$ and $B$ are mutually polar and (4) that $C$ 
and $N$ are polar.

As mentioned in $\S 3, T$ is an involute of $C$; it is described by a point which is fixed on a great circle rolling on $C$. The dual of this construction must lead from $N$ to $B$. By means of the above remarks it is easily seen that $B$ is obtained as the envelope of a family of half great circles emanating from the points of $N$ and being parallel along $N$ in the sense of Levi-Civita's surface parallelism. Analogously $T$ is enveloped by the half great circles obtained by turning those enveloping $B$ through $\pi / 2$ about their initial points on $N$.

Introducing the notation

$$
\sigma(s)=\int_{0}^{s} \omega(s) d s,
$$

we have for the arc lengths of the curves $T, B, N, C$ measured from the points corresponding to $s=0$

$$
\begin{aligned}
s_{T} & =\int_{0}^{s} \kappa d s=\int_{0}^{s} \omega \cos \phi d s, & s_{B} & =\int_{0}^{s} \tau d s=\int_{0}^{s} \omega \sin \phi d s, \\
s_{N} & =\sigma(s), & s_{C} & =\phi(s)-\phi(0),
\end{aligned}
$$

and for their geodesic curvatures (with respect to the sphere)

$$
\begin{array}{ll}
\gamma_{T}=\tan \phi, & \gamma_{B}=\cot \phi, \\
\gamma_{N}=\frac{\phi^{\prime}}{\omega^{\prime}}=\frac{d \phi}{d \sigma}, & \gamma_{C}=\frac{\sigma^{\prime}}{\phi^{\prime}}=\frac{d \sigma}{d \phi} .
\end{array}
$$

A $\kappa$-inflection of $K$ [where $\phi$ passes one of the values $\phi \equiv \pi / 2(\bmod \pi)]$ corresponds to a cusp of $T$ and to an inflection of $B$. A $\tau$-inflection of $K[$ where $\phi$ passes one of the values $\phi \equiv 0(\bmod \pi)]$ corresponds to an inflection of $T$ and to a cusp of $B$. The curve $N$ has no cusps and $C$ has no inflections $(\omega>0)$. Inflections of $N$ and cusps of $C$ do not correspond to singularities of $K$. At these points $\phi$, hence $\tau / \kappa$, is stationary and $K$ behaves locally like a helix.

5. It follows from the Frenet formulae that there exists one and (up to a rigid motion) only one curve $K$ with prescribed functions $\kappa(s)$ and $\tau(s)$ [or $\omega(s)$ and $\phi(s)]$. As a main problem of the differential geometry of closed curves I should like to formulate: To find necessary and sufficient conditions for the curvature and the torsion as periodic functions of $s$ with period $l$ in order that the curve be closed. Probably there is no simple answer to this problem, and so far only a few necessary conditions are known.

However, the problem splits up in a natural way. The Frenet sys- 
tem of differential equations determines the indicatrices $T, B, N$, and from $T$ the curve $K$ itself is found by a simple integration

$$
r=\int_{0}^{s} t d s=\int_{0}^{s T}(1 / \kappa) t d s_{T} .
$$

Instead of the arc length $s$ of $K$ we introduce as a new parameter the arc length $\sigma=s_{N}$ of $N$, which is an increasing function of $s$. Then the Frenet system becomes

$$
\begin{array}{ll}
\frac{d t}{d \sigma}= & n \cos \phi \\
\frac{d n}{d \sigma}=-t \cos \phi & +b \sin \phi \\
\frac{d b}{d \sigma}= & -n \sin \phi
\end{array}
$$

which shows that the indicatrices are already determined if the single function $\phi=\phi(\sigma)$ is given. Since (8) yields

$$
\phi(\sigma)=\phi(0)+\int_{0}^{\sigma} \gamma_{N} d \sigma
$$

we see that $T, B, N$ are determined if a constant $\phi(0)$ and the geodesic curvature $\gamma_{N}$ of $N$ as a function of the arc length of $N$ are given. Elimination of $t$ and $b$ from (10) yields the equation

$$
\frac{d^{2} n}{d \sigma^{2}}=-n+\gamma_{N} n \times \frac{d n}{d \sigma}
$$

which determines the curve $N$ (up to a rotation about $O$ ) when its geodesic curvature $\gamma_{N}$ is given as a function of the arc length $\sigma$.

Summarizing we are led to the following problems:

I. To find conditions which a periodic function $\gamma_{N}(\sigma)$ must satisfy in order that the spherical curve for which $\sigma$ is the arc length and $\gamma_{N}$ the geodesic curvature be closed.

No contributions to this problem seem to exist. The analogous problem for plane curves is trivial since the differential equation corresponding to (12) can be solved explicitly in terms of simple integrals.

II. To find conditions which a closed spherical curve must satisfy in order that it be: (1) the indicatrix of the tangents; (2) the indicatrix of the binormals; (3) the indicatrix of the principal normals of a 
closed space curve.

We are going to discuss this last problem, which is considerably more elementary than I. For, from the geometrical relations between the indicatrices described in $\$ 4$ we get

$$
t=-\frac{d n}{d \sigma} \cos \phi+n \times \frac{d n}{d \sigma} \sin \phi, t=b \times \frac{d b}{d s_{B}} .
$$

Together with (9) this shows that, given any one of the indicatrices $T, B, N$, the curve $K$ can be obtained by the simple integrations (9), (11), a fact which, of course, has been known for a long timè [13].

6. A necessary condition which the tangent indicatrix $T$ of a closed curve $K$ must satisfy is easily derived. Consider any plane which does not intersect $K$. Then the tangents at those points of $K$ which have maximal and minimal distance from the plane are parallel to that plane. Transferred to the unit sphere this means that $T$ is intersected (at least twice) by every great circle, ${ }^{2}$ in other words, the center $O$ of the unit sphere is an inner point of the convex hull to $T$. This condition has also been shown to be sufficient $[6 ; 17 ; 9]$. If it is satisfied, there exists a great variety of closed curves $K$ which have the given tangent indicatrix $T$. These curves have everywhere positive curvature if $T$ has no cusps.

From the necessity of this condition several conclusions may be drawn. The "absolute length" $\int\left|d s_{T}\right|$ is greater than or equal to $2 \pi$. Hence, for the total absolute curvature of a closed curve $K$ with length $l$ we have

$$
\int_{0}^{l}|\kappa| d s \geqq 2 \pi
$$

This has been proved in different ways $[5 ; 11 ; 15 ; 3 ; 4]$. In $\mathrm{B}$. Segre's proof (refound by Rutishauser and Samelson [12]) it is deduced from the following lemma: A spherical curve with length less than or equal to $2 \delta<2 \pi$ is contained in a circle with spherical diameter $\delta$. I mention that (13) also follows from a formula which is proved in integral geometry $[2$, p. 81]: Let $L$ be the (absolute) length of a spherical curve and let $n(x)$ be the number of points in which the great circle with the pole $x$ intersects the curve, then

$$
L=\frac{1}{4} \int n(x) d \alpha
$$

where $d \alpha$ denotes the area element described by $x$ and where the

${ }^{2}$ As far as I know this remark is due to C. Loewner (see [5]). 
integration is extended over the whole sphere. According to our condition for $T$ we here have $n \geqq 2$.

By means of Schwarz's inequality we obtain from (13)

$$
(2 \pi)^{2} \leqq\left(\int_{0}^{l}|\kappa| d s\right)^{2} \leqq l \int_{0}^{l} \kappa^{2} d s,
$$

hence for the length $l$ of an arbitrary closed space curve

$$
l \geqq \frac{2 \pi}{\max |\kappa|}
$$

a theorem due to H. A. Schwarz. ${ }^{3}$

If the class of curves $K$ or the class of spherical curves $T$ considered is restricted, sharper or different results may be obtained.

Answering affirmatively a question by K. Borsuk [3], I. Fáry [4] recently proved that if the curve $K$ is knotted we have

$$
\int_{0}^{l}|\kappa| d s \geqq 4 \pi \text {. }
$$

His proof is based on the interesting remark that the total (absolute) curvature of a space curve $K$ is the mean value of the total (absolute) curvatures of the orthogonal projections of $K$ on the planes through a fixed point. Under our (stronger) assumptions (15) can also be derived from (14). Let $P$ be any plane not intersecting a closed curve $K$ without multiple points. Suppose that the distance from $P$ to a point of $K$ as a function of $s$ has only 2 extremals. Then $K$ may be divided into two arcs such that the distance increases on one of them and decreases on the other. Hence every plane $P^{\prime}$ parallel to $P$ and intersecting $K$ intersects in exactly two different points. Join these points by a segment. Then these segments for all planes $P^{\prime}$ considered make up a surface bounded by $K$ and homeomorphic to a circular disk. Hence $K$ cannot be knotted. For a knot $K$ it follows that the distance of its points from $P$ has at least 4 extremals, that is, at least 4 tangents of $K$ are parallel to an arbitrary plane. This means that every great circle intersects $T$ in at least 4 points. Hence $n \geqq 4$ in (14), which yields (15). ${ }^{4}$

7. We consider now the indicatrix $B$ of the binormals. By means of

${ }^{3}$ For references and related results see $[1, \S 31]$.

- (Added December 1950.) Since this paper was written, important new results concerning the total curvature of knots have been published: J. W. Milnor, On the total curvature of knots; R. H. Fox, On the total curvature of some tame knots, Ann. of Math. vol. 52 (1950) pp. 248-257, 258-260. 
the spherical polarity in which $T$ and $B$ correspond to each other we get at once as a necessary and sufficient condition that a closed spherical curve $B$ be the binormal indicatrix of a closed curve $K$, the following: the tangent great circles of $B$ cover the whole sphere, that is, from every point of the sphere a tangent great circle to $B$ can be drawn.

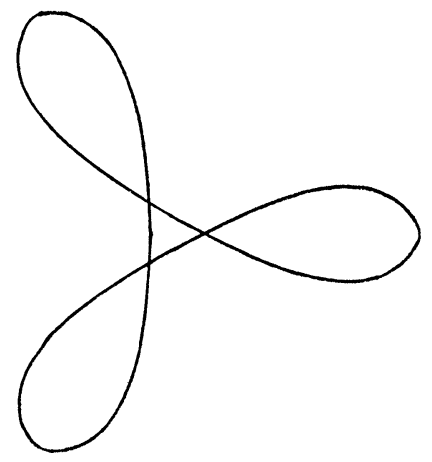

FIG. 2

Of course, there is no positive lower bound for the total (absolute) torsion

$$
\int_{0}^{l}|\tau| d s=\int\left|d s_{B}\right|
$$

of $K$, that is, the absolute length of $B$. But even if we assume that $\kappa$ and $\tau$ are positive at all points of $K$, that is, that $B$ has neither inflections nor cusps, there is no such bound. For, a small spherical curve of the shape sketched in Fig. 2 has these properties and its tangent great circles obviously cover the whole sphere. Hence there exist closed curves with everywhere positive curvature and everywhere positive torsion whose total torsions are arbitrarily small. The study of the example of Fig. 2 seems to indicate that if $\kappa>0$ and $\tau>0$ are assumed there may be a lower bound greater than $2 \pi$ for the sum of the total curvature and the total torsion.

From his lemma mentioned in $\$ 6$, B. Segre [16] has derived an estimate for the total absolute torsion. If $B$ is contained in a hemisphere, let $\delta$ be the spherical diameter of the circumscribed circle of $B$; put $\delta=\pi$ otherwise. Then

$$
\int_{0}^{l}|\tau| d s>2 \delta
$$


That this inequality is sharp for any given $\delta$ (even under the restrictions $\kappa>0, \tau>0$ ) follows from the fact that a spherical curve $B$ of the shape sketched in Fig. 3 satisfies our conditions and may have a length which deviates arbitrarily little from $2 \delta$.

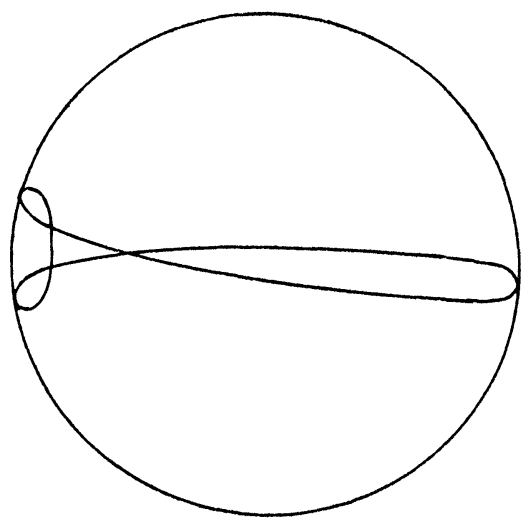

Fig. 3

8. Finally we consider the indicatrix $N$ of the principal normals and, at the same time, its polar, the centrode $C$. Here a necessary condition results from the periodicity of $\kappa$ and $\tau$ which implies

$$
\phi(l)-\phi(0)=2 \pi \nu
$$

for some integer $\nu$, which is called the nutation of the curve $K$ (W. Scherrer [14]). Hence, we conclude from (6) that the total length of the centrode $C$ is $2 \pi \nu$. This is just the condition that every point of a great circle rolling on $C$ describes a closed curve, in other words, that the involutes of $C$, hence $T$ and $B$, are closed. For the curve $N$ we get from (8)

$$
\int_{0}^{l_{N}} \gamma_{N} d \sigma=2 \pi \nu .
$$

This means that if a tangent vector to the sphere at a point of $N$ is displaced along $N$ by Levi-Civita's surface parallelism it will return to its initial position after the curve has been traversed once. We see again that (16) implies that $T$ and $B$ are closed, and conversely.

An immediate consequence of $(16)$ is that $\phi(s)$ for $0 \leqq s \leqq l$ passes a value congruent to $\pi / 2(\bmod \pi)$ at least $2 \nu$ times and likewise a value congruent to $0(\bmod \pi)$ at least $2 \nu$ times. Hence we have:

A closed space curve with the nutation $\nu$ has at least $2 \nu k$-inflections and at least $2 \nu \tau$-inflections. 
If $\kappa>0$ is assumed, we have $\nu=0$. If, in addition, $N$ has no multiple points, (17) and the Gauss-Bonnet formula yield Jacobi's theorem $[8 ; 7]$ : The indicatrix of the principal normals of a closed space curve bisects the surface of the unit sphere. The connection of this theorem with his concept of parallelism has been observed by $T$. Levi-Civita [10], and the more general form (17) has been stated by W. Scherrer $[14] .{ }^{5}$

In order to discuss the question whether condition (17) for $N$ is sufficient I recall that if $N$ is given, $T$ (and $B$ ) is obtained as the envelope of a system of half great circles which are parallel along $N$. The direction of one of these circles may be prescribed arbitrarily according to the choice of the constant $\phi(0), 0 \leqq \phi(0)<2 \pi$. Hence there is a one-parameter family of curves each of which may be taken as $T$ or $B$ if it satisfies the corresponding condition discussed in $\$ \S 6$ and 7 respectively. Consequently the condition (17) for $N$ will be sufficient if the following statement is true: For every closed spherical curve satisfying (17) at least one of these envelopes is intersected by every great circle; or (formulated for $B$ ) for every such curve a family of great circles exists which covers the whole sphere and is obtained from one of the great circles by parallel displacement along $N$.

I conclude my lecture with this open question. I hope that I have been able to show that the differential geometry of closed curves, though elementary, comprises interesting problems.

\section{REFERENCES}

1. W. Blaschke, Vorlesungen über Differentialgeometrie I, 3d. ed., Berlin, 1929; New York, 1945.

2. - Vorlesungen ilber Integralgeometrie II, Leipzig and Berlin, 1937; New York, 1950.

3. K. Borsuk, Sur la courbure totale des courbes fermées, Annales de la Société Polonaise de Mathématique vol. 20 (1948) pp. 251-265.

4. I. Fáry, Sur la courbure totale d'une courbe gauche faisant un noud, Bull. Soc. Math. France vol. 77 (1949) pp. 128-138.

5. W. Fenchel, Über Krilmmung und Windung geschlossener Raumkurven, Math. Ann. vol. 101 (1929) pp. 238-252.

${ }^{5}$ Scherrer's argument and his example showing that $\nu \neq 0$ may occur are in accordance with our assumptions, but are inconsistent with his own which exclude $\kappa$-inflections.

Scherrer aims at a formulation of (17) in terms of the spherical area bounded by $N$. To this purpose he gives a definition of the area bounded by a spherical curve with multiple points. However, this definition seems artificial and is open to objections. Compare S. B. Jackson's review of this paper [Mathematical Reviews vol. 8 (1947) p. 226]. A natural relation, the dual of (14), between the integral of the geodesic curvature and the areas of the regions into which the sphere is divided by the curve and its opposite curve is given by Blaschke [2, p. 83]. 
6. - Geschlossene Raumkurven mit vorgeschriebenem Tangentenbild, Jber. Deutschen Math. Verein. vol. 39 (1930) pp. 183-185.

7. - Über einen Jacobischen Satz der Kurventheorie, Tôhoku Math. J. vol. 39 (1934) pp. 95-97.

8. C. G. J. Jacobi, Über einige merkwilrdige Curventheoreme, Astronomische Nachrichten vol. 20 (1842) pp. 115-120; Gesammelte Werke, vol. 7, pp. 34-39.

9. M. Krein, Sur un theorème de Vigodsky, Rec. Math. (Mat. Sbornik) N.S. vol. 18 (1946) pp. 447-450.

10. T. Levi-Civita, Curve chiuse a parallelismo monodromo sopra la sfera, Pontificia Academia Scientiarum Acta vol. 87 (1934) 10 pp.

11. H. Liebmann, Elementarer Beweis des Fenchelschen Satzes ïber die Krilmmung geschlossener Raumkurven, Preuss. Akad. Wiss. Sitzungsber. (1929) pp. 292-293.

12. $\mathrm{H}$. Rutishauser and $\mathrm{H}$. Samuelson, Sur le rayon d'une sphère dont la surface contient une courbe fermée, C. R. Acad. Sci. Paris vol. 227 (1948) pp. 755-757.

13. G. Scheffers, Anwendung der Differential- und Integralrechnung auf Geometrie, vol. 1, Einfilhrung in die Theorie der Curven in der Ebene und im Raume, Leipzig, 1901, pp. 240-251.

14. W. Scherrer, Über das Hauptnormalenbild einer Raumkurve, Comment. Math. Helv. vol. 19 (1947) pp. 115-133.

15. B. Segre, Sui circoli geodetici di una superficie a curvatura totale costante, che contengono nell'interno una linea assegnata, Bollettino della Unione Matematica Italiana vol. 13 (1934) pp. 279-283.

16. - - Sulla torsione integrale delle curve chiuse sghembe, Atti della Accademia Nazionale dei Lincei. Rendiconti. Classe di Scienze Fisiche, Mathematiche e Naturali (8) vol. 3 (1947) pp. 422-426.

17. M. Vigodsky, Sur les courbes fermées d indicatrice des tangentes donnee, Rec. Math. (Mat. Sbornik) N.S. vol. 16 (1945) pp. 73-80.

The University of Southern California 